Revue d'études américaines. American Studies Journal

\title{
William A. Gleason, Sites Unseen. Architecture, Race and American Literature
}

Michel Imbert

\section{(2) OpenEdition}

\section{Journals}

Electronic version

URL: https://journals.openedition.org/transatlantica/7097

DOI: 10.4000/transatlantica.7097

ISSN: 1765-2766

\section{Publisher}

Association française d'Etudes Américaines (AFEA)

\section{Electronic reference}

Michel Imbert, "William A. Gleason, Sites Unseen. Architecture, Race and American Literature",

Transatlantica [Online], 2 | 2014, Online since 05 March 2015, connection on 06 February 2023. URL:

http://journals.openedition.org/transatlantica/7097 ; DOl: https://doi.org/10.4000/transatlantica.7097

This text was automatically generated on 6 February 2023.

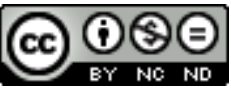

Creative Commons - Attribution-NonCommercial-NoDerivatives 4.0 International - CC BY-NC-ND 4.0

https://creativecommons.org/licenses/by-nc-nd/4.0/ 


\title{
William A. Gleason, Sites Unseen. Architecture, Race and American Literature
}

\author{
Michel Imbert
}

\section{REFERENCES}

GLEASON, William A., Sites Unseen. Architecture, Race and American Literature, New York, New York University Press, 2011, ISBN : 9780814732472, 288 pages, 37 illustrations, $19 € / 13 \$$

1 William Gleason dresse un état des lieux de la représentation littéraire de l'habitat nord-américain tout au long du XIXe siècle. Il tente de décrypter l'architecture des récits, au double sens objectif et subjectif du génitif. Il s'interroge à la fois sur l'agencement intérieur du cadre où se déroule le récit pour mettre au jour son fondement caché et sur la structure sous-jacente du récit perçue également comme une construction symbolique. A l'évidence, les intérieurs américains portent l'empreinte des femmes, qui jouent un rôle déterminant dans l'économie domestique des foyers de l'Amérique victorienne, mais la répartition sociale des places au sein du Home est également fonction de la couleur de la peau ou de l'origine ethnique. C'est ainsi que la véranda si typique des maisons du Sud est le théâtre d'une lutte insidieuse entre maîtres blancs et serviteurs noirs dans les contes de chesnutt, tantôt parce que l'élite noire émergente édifie des demeures agrémentées d'un portique sur le modèle de celles des anciens maîtres, au risque de devenir la cible du Ku Klux Klan, tantôt parce que les domestiques qui étaient des esclaves occupent symboliquement le terrain. Gleason montre de façon convaincante qu'il n'est pas indifférent que le narrateur noir des Conjure Tales s'exprime sur les marches du perron ou qu'il côtoie le maître de maison sur la véranda; il est plus significatif encore qu'il surprenne le prétendu maître en surgissant de l'intérieur comme s'il était en pays conquis. Fait révélateur, chacun des contes enchâssés s'ouvre sur cette galerie qui encadre le récit et relie en puissance 
l'intérieur et l'extérieur, la demeure des maîtres et les dépendances où sont cantonnés les esclaves affranchis devenus domestiques. Comme dans le récit liminaire des Piazza Tales d'Herman Melville, l'économie du récit, au propre et au figuré, en dépend. Dans les récits de Chestnutt, la structure de l'intrigue s'articule sur le plan de la maison coloniale. Comme dans The House of the Seven Gables, la maison au double sens de lignée et d'habitat est la scène à la fois invariable et tournante où les rapports de force entre dominants et dominés peuvent toujours s'inverser. Comme dans la romance de Hawthorne, ce sont des histoires de possession matérielle et mentale, autrement dit de spoliation et d'aliénation réciproques: une ancienne esclave dont la langue a été tranchée est la seule à connaître la cachette où est dissimulé le titre de propriété recherché par le maitre cruel qui a été autrefois son amant; elle se garde de lui communiquer l'accès au titre convoité qui est dissimulé dans le rocking-chair qu'elle occupe sur la véranda et d'où elle observe la chute de ceux qui sont responsables de ses malheurs.

2 Le premier chapitre est consacré au premier roman d'une esclave, The Bondwoman's Narrative d'Hannah Crafts, un genre d'autofiction avant la lettre. L'esclave en cavale rêve d'un chez-soi, d'un modeste « cottage » qui lui appartiendrait plutôt qu'une de ces belles demeures patriciennes entachées par les horreurs de l'esclavage, non seulement l'exploitation généralisée qui est à l'origine de la fortune des planteurs mais les crimes d'un autre ordre commis dans l'enceinte même de la maison coloniale : la maîtresse des lieux doit s'accommoder non sans mal des amours ancillaires du maitre qui dissimulent à peine une forme d'esclavage sexuel. Pour une jeune femme noire, exposée au harcèlement sexuel, le rêve d'intimité et d'accession à la propriété se double en fait de crainte continuelle du viol. William Gleason souligne les similitudes entre l'héroïne du roman et celle de Bleak House, promise à son tuteur mais assez magnanime pour qu'il la laisse épouser un autre et lui offre un cottage. Ne plus être un bien meuble, la " chose " du maitre ou sa domestique à tout faire mais avoir une maison à soi, tel est le rêve commun des gouvernantes anglaises et des esclaves noires dans l'Amérique d'avant la Guerre de Sécession. Les pages empruntées à Bleak House ne sont pas purement plagiées; elles sont transposées de l'East End dans le Sud profond. L'étude détaillée de cette transformation par translation de Bleak House en «Black House » est particulièrement fine.

3 Le second chapitre, consacré à Chestnutt, retrace l'évolution de la lutte des classes et des races dans l'Amérique de la Reconstruction et du tournant du siècle. Nous n'y revenons pas. L'horizon s'élargit à partir du quatrième chapitre puisqu'il n'est plus circonscrit à l'architecture coloniale du Sud profond: en effet, après les grandes demeures (mansions) et les maisons de campagne (cottages) calquées sur le modèle anglais mais importées, acclimatées sur le sol américain, Gleason aborde le bungalow en kit que la firme Aladdin exporte dans le monde entier et plus particulièrement en Amérique latine. La portée de l'essai prend de l'extension et s'étend à l'hémisphère sud, au continent latino-américain dans le droit fil des «hemispheric studies ». Cette fois, Gleason confronte deux récits de voyages: celui d'un Américain qui observe avec bienveillance l'expansion de l'empire Sud dans la zone sud et réciproquement, le témoignage autobiographique d'une jeune rescapée de la révolution mexicaine réfugiée dans le Texas.

4 Après avoir abordé l'axe Nord-Sud du point de vue de l'architecture intérieure, le quatrième chapitre traite des rapports entre le Wild West et l'Extrême Orient à l'ère du 
péril jaune. Mêlant high and low culture, Gleason confronte des romans policiers populaires qui ont pour cadre Chinatown et l'orientalisme marqué perceptible dans l'architecture d'un Frank Lloyd Wright pratiquement à la même époque. L'image de l'Extrême-Orient qui se dégage de cette comparaison est complexe, voire contradictoire, tandis que le Chinatown de la pulp fiction est un bazar oriental encombré de bibelots et un dédale où le détective blanc risquerait de se perdre sans le secours d'un complice chinois. Au contraire, Frank Lloyd Wright, impressionné par la reconstitution d'un temple japonais lors de l'exposition mondiale de Chicago, dessine ses maisons dans la prairie sur le modèle nippon: il supprime autant que possible les murs qui confinent et permet le passage fluide entre l'intérieur et le milieu environnant. Comme les chapitres précédents, l'étude repose sur une logique dialectique nullement réductrice. De même que le rêve de propriété était habité par le spectre de la possession sexuelle synonyme de viol de l'intimité chez Hannah Crafts comme chez Chestnutt, de même c'est ce mélange d'attraction et répulsion qui caractérise les relations entre l'empire américain à vocation planétaire et l'afflux d'immigrants asiatiques. Hawaï est le théâtre de cette confrontation ambiguë.

5 En guise d'épilogue, l'essai revient sur les mutations de la Maison Blanche depuis sa construction jusqu'à ce qu'elle devienne l'emblème de l'accession au pouvoir du premier président noir des Etats-Unis : sa couleur hautement symbolique et son vaste portique surmonté d'un tympan néo-classique apparentent ce haut lieu de la première démocratie moderne à l'architecture impériale ou coloniale, comme si l'architecture trahissait sa vocation expansionniste et pas seulement la vertu républicaine héritées de l'Athènes ou de la Rome antiques. Il est significatif que dans ce lieu rebaptisé Maison Blanche sous Theodore Roosevelt, où s'affiche la suprématie de la race blanche à travers sa façade unicolore, la différenciation des fonctionnaires suivant leur origine raciale au sein de l'édifice national reflète l'évolution de la discrimination sur l'ensemble du territoire: les fonctionnaires noirs partageaient leur repas avec leur homologues blancs avant que les règles discriminatrices ne se mettent en place, mais la hiérarchie des catégories professionnelles s'est doublée d'une ségrégation stricte en fonction de la race sous la présidence.

6 Comme les travaux de Dell Upton, Sites Unseen entrelace l'étude de l'architecture vernaculaire et l'histoire sociale; il apporte un éclairage tout à fait original au sujet de l'empreinte des relations entre les races sur le paysage architectural. Il renouvelle le regard critique sur des récits comme ceux de Charles Chesnutt. On ne saurait trop recommander la lecture de cet essai novateur à la croisée des disciplines.

\section{AUTHORS}

MICHEL IMBERT

Université Paris Diderot 